\title{
Ideographic Distinctions Between Methamphetamine Induced Persistent Psychosis and Schizophrenic Disorders: A Qualitative Clinical Analysis Toward Differential Diagnosis
}

\author{
Reuben Vaisman-Tzachor* \\ California Licensed Psychologist, Counseling Center of Santa Monica, USA
}

*Corresponding author: Reuben Vaisman-Tzachor, California Licensed Psychologist 15091, Counseling Center of Santa Monica - A Psychological Corporation, 1731 Barry Avenue, \# 112, Los Angeles, California 90025, USA.

Received Date: February 11, 2019

Published Date: April 02, 2019

\begin{abstract}
Clinical presentations of Methamphetamine induced persistent psychosis and Schizophrenic disorders appear similar, causing diagnostic uncertainty and treatment difficulties. The two conditions differ in responses to introduction of anti-psychotic medicines, versus anti-seizure and anti-depressant medicines; they differ in responses to psychotherapy, and they differ in their prognoses. This article makes descriptive distinctions between the conditions that offer differential diagnoses, divergent treatment directions, and calls for future research.
\end{abstract}

Keywords: Methamphetamine induced persistent psychosis; Schizophrenic disorders

\section{Introduction}

Traditional theories of abnormal psychology generally consider schizophrenic disorders a unique and distinct group within the larger category of psychotic disorders. The DSM-5 APA [1], further distinguishes the various schizophrenic disorders from other psychotic conditions based largely on their most salient corresponding presentations. For example, schizophrenic disorder, paranoid type is primarily distinguished from other conditions within this group (or other disorders for that matter), by the presence of persistent paranoid thoughts and beliefs. These are usually accompanied by commensurate distrustful attitudes, anxious feelings, and defensive behaviors.

Over the years, numerous attempts at defining particular etiologies that could explain the emergence of these conditions, usually in late adolescence or early adulthood of lives of relatively small percent (estimates run between $1 \%-3 \%$ ) of humans failed to yield substantial results. Most researchers and practitioners nowadays commonly consider factors such as inherent biological "vulnerabilities" or "pre-dispositions" (such as having biological relatives with the disorder) and environmental stressors, which when combined in the life of individuals, could give rise and full expression to the schizophrenic disorder [2-4]. Not surprisingly though, the co-morbidity rates of Schizophrenia with substancerelated disorders are also high, leading to potential problems with differential diagnosis [1].

What most professionals also seem to agree upon is that whatever the reason, the most critical elements in the biology of schizophrenic disorders is found in the malfunction of neural communication in the brain. Specifically, the malfunction seems to concentrate primarily in neurons whose communication is facilitated by the chemical neurotransmitter called Dopamine. While nerves that utilize Dopamine are involved in many cortical and sub-cortical mental activities, they seem to concentrate in communication of thinking, sensory, motor, and emotional mental processes. The malfunction of Dopamine nerve communication in schizophrenics appears to be rooted in the development of over-zealous activity of neurons which utilize Dopamine. This development in-turn, results in sensory, mental, and emotional activity which largely exceeds normal human experience [2,5]. 
The excess mental activity of Dopamine nerves is largely considered to be responsible for the primary symptoms of schizophrenic disorders involving hallucinations (internally generated sensory experiences which do not correspond to external reality); delusions (attitudes and beliefs that are grossly outside the human norm and which defy common sense); serious problems with thinking (difficulty in maintaining coherent sequence of speech and logical thinking), and movement disorders (either repetitive movements or lack of movement for extended periods of time). As such, these symptoms are regarded as "positive symptoms" in that they add to the normal mental activity of most humans, additional layers of sensory, mental, and emotional experiences that are excessive, grossly disruptive to the person's normal mental activity, and severely disturbing to the individual [6].

Most persons diagnosed with schizophrenia this author interviewed over three decades of clinical work described their experiences with the disease as unsettling and terrifying. Many called their hallucinations "horrific nightmares" (because of the gross distortions of sensory experiences); their delusions "isolating" (because their beliefs others did not share), and their thinking problems "very frustrating" (because they impinged upon functioning and communication with others). Thus, the term of arts ascribed to these patients is accurate in that they indeed subjectively "suffer" from schizophrenia.

Not surprisingly though, these patients also develop "negative symptoms" involving largely reduction in what is considered "normal" mental, social, and intellectual activity. This phenomena is often described as diminished social interaction (for fear of rejection and ridicule, communicating in a monotone voice with little facial expression); concomitant depression (e.g., feeling inadequate or inferior to others, feeling sadness); motor inertness (difficulty in initiation and execution of daily activities), and diminished mental activity noted mostly in poverty of speech and rare engagement in voluntary thoughtful processes and problem solving [1,2].

\section{Medicinal Treatment of Schizophrenic Disorders}

Consequent to the aforementioned, most if not all of the anti-psychotic medicines that were developed over the years sought to address this precise effect. Namely, to counteract the abnormally active Dopamine-mediated neural paths in order to reduce the symptoms of psychosis. Not surprisingly, all medicines which reduce the activity of Dopamine-mediated neural paths in psychotic patients indeed manage to reduce the expression of "positive symptoms" of psychosis (i.e., hallucinations, delusions, thought disorder, movement disorder) in those afflicted with these conditions. What has also become apparent in the years of clinical use of anti-psychotic medicines of the Dopaminemediated paths, is that whatever "negative symptoms" (i.e., social withdrawal, depression, poverty of thought, etc.) there were before administration, those remained in effect or became worse [7].

The common explanation accepted by most is that Dopaminemediated neural paths enervate also circuits involved in problemsolving mental processes (in pre-frontal cortical regions of the brain), and circuits involved in the experience of pleasure (from social interaction, for instance) of the limbic system. Once these neural paths are rendered less active by the introduction of anti-psychotic medicines, the person becomes more prone to depression, more socially withdrawn, and sometimes reaches extremes of catatonic states of motor inertia [8]. These unfortunate side-effects often lead to patients' discontinuation of medicinal treatment because the negative symptoms are subjectively painful and not well tolerated over time.

\section{Methamphetamine Induced Persistent Psychosis}

The presentation of Methamphetamine induced persistent psychosistends to mimicthe presentation of schizophrenic disorders in that the salient elements become evident after prolonged exposure to Methamphetamine. Characteristically, the chronic Methamphetamine user begins to display psychotic symptoms akin to the "positive symptoms" of psychosis (i.e., hallucinations, delusions, thought disorder) along with other physical symptoms (e.g., loss of hair, loss of teeth, skin tissue deterioration, etc.). Unlike the case with schizophrenic disorders, "negative symptoms" of psychosis (e.g., social withdrawal, depression, etc.) do not seem apparent in the chronic Methamphetamine user who also displays "positive symptoms" of psychosis [1].

The commonly held presumption about persons whose drug of choice is Methamphetamine is that underlying this choice of a stimulant is a psychological character makeup which naturally tends to depression and avoidance of problems. While the background narrative of how the person began to use the drug may involve other themes (e.g., the person was introduced to the drug in a party), the appeal of the stimulant is what sustains the use of the substance over time. Hence, the stimulant serves to both "medicate away" depressive feelings and to avoid dealing with problems that appear to the individual insurmountable, and therefore the use of Methamphetamine persists [2].

Most clinicians and researchers believe that the stimulating effects on the brain resulting from ongoing exposure to Methamphetamine are responsible for masking the depressing effects of the psychotic process. Others argue that the stimulating effects of Methamphetamine on the brain essentially prevent whatever "negative symptoms" of psychosis that may exist from becoming expressed altogether. Both these perspectives seem to be at least partially supported, and the substantiating evidence comes from clinical work with Methamphetamine addicts who by and large, admit that the use of a stimulant drug was initiated to "medicate away" impending depression. Hence, the chronic use of Methamphetamine is largely designed to ward off painful depressive feelings and devastating depressed mood, which also either masks or eliminates altogether these symptoms from the person's presentation $[1,3]$.

Thus, at first flush, the florid schizophrenic patient and the patient exhibiting Methamphetamine induced persistent psychosis appear to be the same. In both, the most prominent symptoms of psychosis are the positive kind, involving some combination variants 
of hallucinations, delusions, and severely disturbed thinking. These more obvious symptoms which are also more disruptive and requiring immediate attention and urgent interventions also mask the other, negative symptoms of psychosis that are lurking underneath the surface [8].

\section{Differences Emerge During Treatment}

The primary differences between the two conditions emerge during the course of treatment, which often involve a combination of psychiatric medicines prescribed in conjunction with psychotherapy or counseling. The psychiatric protocol usually calls for dramatic intervention at the acute states of treatment with intra-muscular injections of a potent anti-psychotic agent (e.g., Haloperidol), which is then tapered off within a few days. Concurrent with tapering off first-line anti-psychotic medicine the protocol calls for the introduction of other, orally administered anti-psychotic medicines (e.g., Buspirone, Lamotrigine, Quetiapine, etc.). Simultaneous with the introduction of oral anti-psychotic medicines the treatment protocol ordinarily calls for psychological intervention in the form of intensive psychotherapy [7].

Patients who undergo some variation of this intervention protocol and who entered treatment for a psychotic disorder of a schizophrenic type will likely see improvement in the gradual and progressive diminution of "positive symptoms." They will exhibit substantially lesser hallucinations, endorse lessened delusional beliefs, and their speech will evince greater clarity of thought and overall coherence. At the same time, these patients will become more receptive to psychological treatment vis-a-vis psychotherapeutic interventions. This will be evidenced in the persons' expression of hope, the desire to effect changes in their lives and the wish to make changes to the ways in which they conduct themselves socially. The communication with care givers will become more linear and coherent, and there would be a decreased need for speculation about the true intentions and / or needs of the patient [9].

Following an initial period of complete recovery, there would begin to emerge secondarily the "negative symptoms" of psychosis, involving lethargy, low mood, inertia, boredom, and general malaise. Those would be then addressed by second order psychiatric medicines of the anti-depressant and / or anti-convulsive types combined with psychological interventions focused on forestalling an impending depressive decline. Ordinarily this approach obtains the majority of the therapeutic goals which substantially diminish "positive symptoms" of psychosis and prevent a slip to depression and expression of secondary "negative symptoms" of psychosis [8].

\section{Methamphetamine Users' Response to Medicinal Treatment}

The response of the person whose psychotic symptoms are largely believed to be induced by the long-term effects of chronic Methamphetamine use is of an entirely different trajectory. While the introduction of first line of anti-psychotic medicines produces characteristic heavy sedation in both populations, subsequent attempts to substantially reduce "positive symptoms" of psychosis in the stimulant induced condition is met with only mild or insignificant improvement. That means that the introduction of orally administered, second-order anti-psychotic medicines produces only small reduction of hallucinations, delusions, and problems with thinking in the Methamphetamine induced (hence, persistent) psychosis [3].

In many drug-addiction rehabilitative programs the common protocol following medical detoxification involves the introduction of anti-seizure medicines (most frequently Gabapentin or Topiramate) which seem to generally better dampen the emotional exuberance and mental disorganization left behind Methamphetamine chronic use. That is usually done because regular anti-psychotic medicines cannot temper the "positive symptoms" of psychosis of persons whose history involved regular use of Methamphetamine. It is important to note that the efficacy of anti-seizure medicines in reducing "positive symptoms" of psychosis is largely rated only to "just below tolerable" levels for the patient and for those in direct contact with them [10].

Concomitant with completion of medical detoxification, intensive psychological interventions in the form of psychotherapy and counseling are also called for in the common rehabilitative treatment protocols. These are largely directed at managing the urges to return to use stimulating drugs, and to learn to manage life under the new circumstances in the person's life. These circumstances often entail ways to live with an irreversibly changed brain and seriously reduced mental faculties. For the person who is in rehabilitative treatment the choices are often unpalatable - either to live with diminished mental capacity or to continue to use drugs to avoid dealing with the devastation already wrecked upon the self. Hence, psychological treatments for persons who suffer from Methamphetamine induced persistent psychosis are only modestly successful in helping patients accept themselves in their now, lesser states and many revert back to previous drug user life-style $[3,8]$.

Interestingly, psychiatric interventions with persons whose psychosis is believed to have been induced by chronic use of Methamphetamine tend to exhibit much less pronounced "negative symptoms" of psychosis. This may be attributable to the removal of or the reduction of anti-psychotic medicines which are suspect in increasing or facilitating the emergence of subsequent "negative symptoms" in the course of the treatment. This effect may also be attributable to generally better effects anti-seizure medicines commonly used in this protocol have in controlling depressive symptoms. This may be so because depressive symptoms closely resemble "negative symptoms" of psychosis and could be reduced by introduction of anti-seizure medicines [11].

\section{Explanations for the Difference in Response to Medicinal Intervention}

There are numerous theories attempting to explain the divergent responses to medicinal treatments that persons suffering from Methamphetamine induced persistent psychosis exhibit, and the poor prognosis for this group. They all seem to point at neuro-degeneration in the brains of persons chronically using 
Methamphetamine as the primary cause. Neuro-degeneration results from increased likelihood of mechanical injuries of persons under the influence of Methamphetamine which lead to traumatic brain injuries (TBI). Additionally, the physiological side-effects on the circulatory system of persons chronically using Methamphetamine often produces ischemic damage such as stroke and / or brain aneurism. Finally, the toxic effects that activate the brain of chronic Methamphetamine users is also implicated in brain-cell necrosis and degeneration rates much greater than typical for other central nervous system diseases (e.g., Alzheimer's; Multiple Sclerosis, Parkinson's, etc.) $[10,11]$.

From a psychological perspective, persons who initially were attracted to use of stimulants such as Methamphetamine may have experienced unfavorable and otherwise depressing conditions in their lives. Consequently, the introduction of a powerful stimulant warded off any genuine feelings of depression and despair in response to the problems these patients were facing. By introducing a powerful stimulant however, these persons also robbed themselves of opportunities to learn to cope with and deal with the unfortunate circumstances of their lives. This in turn, left them more psychologically vulnerable and less prepared to meet the challenges that they were facing compared to others with similar backgrounds. Thus, when temporarily weaned off the stimulant, former Methamphetamine users are now exposed afresh to all of the old dilemmas that they have been avoiding. Furthermore, facing these challenges head on without the adequate skills and coping devices usually make the recovery seem insurmountable, and the alternative to resume drug use much more appealing.

\section{Cannabis Use Complicates the Picture}

The concurrent use of Cannabis by many patients of both categories examined here (i.e., Methamphetamine users and nonusers who are Schizophrenic) complicate the picture and make the distinction efforts more tenuous. This, because Cannabis has been found by numerous studies to be implicated in the occurrence of psychotic symptoms in persons that regularly use it $[12,13]$. In fact, studies indicate that the longer the use of Cannabis, the greater the likelihood for the development of a psychotic disorder $[1,14]$ Similarly, others found that the earlier in age the start of Cannabis use, the greater the likelihood for the development of a psychotic disorder [1,15]. And while others had found some evidence for curative effects of Cannabis use in psychotic patients [16], or general healing benefits to the central nervous system [17], the clinical picture remains inconclusive.

It is a known fact to most clinicians that many Methamphetamine users utilize Cannabis as a "control substance" (i.e., a substance which counteracts the effects of the substance they primarily use, to avoid undesirable effects). Typically, a Methamphetamine user has no idea about the concentration, purity, or dosage of the illicit substance they ingest, since it was manufactured under suspect conditions and without proper regulation or standards of any kind. Learning from experience, many Methamphetamine addicts quickly discovered that the drug they introduced into their body is sometimes much too strong and produces mostly unwanted effects (e.g., intense anxiety, paranoia, rage, intense fear, and physiological discomfort). In order to control the potential aversive effects of Methamphetamine use, most addicts resort to using Cannabis in conjunction with Methamphetamine as an "antidote," for its calming, and anxiety reducing effects. Consequently, the psychotic symptoms observed in chronic Methamphetamine users could be resulting from the concurrent use of Cannabis, or from the mixing of the two drugs together, and not necessarily purely from the use of Methamphetamine [1].

Similarly, it also a known fact to many clinicians that persons who suffer from a Schizophrenic disorder also resort in large numbers to the use of Cannabis for relief form their condition. This, because the Schizophrenic disorder renders patients who suffer from it frequently besieged by frightening hallucinatory experiences that are disorienting and deeply upsetting (e.g., hearing a voice commanding the person to commit suicide). Additionally, patients who suffer from Schizophrenia often develop paranoid delusions which are also frightening and largely debilitating to the person (e.g., believing that the government is pursuing them). They too have therefore learned from experience to sooth and calm themselves with the use of Cannabis as an anti-anxiety agent of sorts. As one patient reported: "When I use Marijuana, I no longer hear my voices, which is all I want to be okay." It is therefore possible that the treatment of persons diagnosed with Schizophrenic disorder may actually be treatment of a psychotic sequella to chronic Cannabis use, and not purely Schizophrenia [1]

\section{Summary of Potentials and Risks}

The clearest benefits from the ideographic distinction between Schizophrenic disorders and Methamphetamine induced persistent psychosis are in the differential diagnosis and divergent psychiatric and psychological treatment directions. A person who responds to the introduction of anti-psychotic medicines with a marked reduction in "positive symptoms" of psychosis would likely benefit from introduction of secondary anti-depressant agents and supportive psychotherapy. The treatment of this population would largely be directed to forestall future psychotic breaks and to maintain the best quality of life possible for the patient. This would be sought, through the patient's acceptance of the Schizophrenic disorder diagnosis and subsequent long-term management with medicines and psychotherapy.

For the person whose response to the introduction of antipsychotic medicines yielded only marginal improvements in the presentation of "positive symptoms" of psychosis, the better option is to then introduce combinations of anti-seizure and anti-depressant medicines. This would be then combined with psychological intervention primarily designed to help the patient maintain a drug-free lifestyle and to engage in rehabilitative efforts. These would likely focus on ameliorating the experience of depression and teaching social and living skills necessary for the patient to cope with their less than fortunate life circumstances. These efforts would be helped greatly by the patient's acceptance 
of their condition being the result of addiction, with commensurate commitment to maintenance of a sober lifestyle.

\section{Acknowledgement}

None.

\section{Conflict of Interest}

Authors declare no conflict of interest.

\section{References}

1. American Psychiatric Association (2013) Diagnostic and Statistical Manual of Mental Disorders ( $5^{\text {th }}$ edn), American Psychiatric Publishing, Washington, USA.

2. NIMH (2019) National Institute of Mental Health.

3. Glasner Edwards S, Mooney LJ (2014) Methamphetamine psychosis: epidemiology and management. CNS Drugs 28(12): 1115-1126.

4. Najjar S, Pearlman DM (2015) Neuroinflammation and white matter pathology in schizophrenia: Systematic review. Schizophr Res 161(1): 102-112.

5. Sass LA (2014) Self-disturbance and schizophrenia: Structure, specificity, pathogenesis (Current issues, New directions). Schizophr Res 152(1): 5-11.

6. Bernstein HG, Steiner J, Guest PC, Dobrowolny H, Bogerts B (2015) Glial cells as key players in schizophrenia pathology: Recent insights and concepts of therapy. Schizophrenia Research 161(1): 4-18.

7. Xue X, Song Y, Yu X, Fan Q, Tang J, et al. (2018) Olanzapine and haloperido for the treatment of acute symptoms of mental disorders induced by amphetamine-type stimulants: A randomized controlled trial. Medicine (Baltimore) 97(8): e9786.
8. Wearne TA, Cornish JL (2018) A Comparison of methamphetamineinduced psychosis and schizophrenia: a review of positive, negative and cognitive symptomatology. Front Psychiatry 9: 491.

9. Parrott AC (2018) Mood fluctuation and psychobiological instability: the same core functions are disrupted by novel psychoactive substances and established recreational drugs. Brain Sci 8(3): e43.

10. Gan H, Zhao Y, Jiang H, Zhu, Y, Chen T, et al. (2018) A research of methamphetamine-induced psychosis in 1430 individuals with methamphetamine use disorder: Clinical features and possible risk factors. Front Psychiatry 9: 551.

11. Chang X, Sun Y, Zhang Y, Muhai J, Lu L, et al. (2018) A Review of risk factors for methamphetamine-related psychiatric symptoms. Front Psychiatry 9: 603.

12. Andrade C (2016) Cannabis and neuropsychiatry: Benefits and risks. J Clin Psychiatry 77(5): e551-554.

13. Gage SH, Hickman M, Zammit S (2016) Association between cannabis and psychosis: Epidemiologic evidence. Biological Psychiatry 79 (7): 549-556.

14. Parakh P, Basu D (2013) Cannabis and psychosis: Have we found the missing links? Asian J Psychiatr 6(4): 281-287.

15. Chadwick B, Miller, M, Hurd YL (2013) Cannabis use during adolescent development: Susceptibility to psychiatric illness. Front Psychiatry 4: 129.

16. Zuardi AW, Crippa JA, Hallak JE, Bhattacharyya S, Atakan Z, et al. (2012) A critical review of the antipsychotic effects of cannabidiol: 30 years of a translational investigation. Curr Pharm Des 18(32): 5131-5140.

17. Scuderi C, Filippis DD, Iuvone T, Blasio A, Steardo A, et al. (2009) Cannabidiol in medicine: A review of its therapeutic potential in CNS disorders. Phytother Res 23(5): 597-602. 